\title{
Effects of surgical facemasks on perceived exertion during submaximal exercise test in healthy children
}

\author{
Gregory Reychler ${ }^{1,2,3,4}$ (D) Marie Standaert ${ }^{4} \cdot$ Nicolas Audag $^{1,2,3,4} \cdot$ Gilles Caty $^{5} \cdot$ Annie Robert $^{6} \cdot$ William Poncin $^{1,2,3,4}$
}

Received: 17 November 2021 / Revised: 23 February 2022 / Accepted: 26 February 2022 / Published online: 3 March 2022

(c) The Author(s), under exclusive licence to Springer-Verlag GmbH Germany, part of Springer Nature 2022

\begin{abstract}
Only a few data associated to wearability of facemask during exercise are available in children. The aim of the study was to evaluate the effect of wearing a facemask on perceived exertion (primary aim), dyspnea, physical performance, and cardiorespiratory response during a submaximal exercise test in children aged between 8 and 12 years. This study was performed in 2021 in healthy volunteer children from 8 to 12 years. They performed prospectively two 1-min sit-to-stand tests (STST), with or without a surgical facemask. The perceived exertion (modified Borg scale), dyspnea (Dalhousie scale), heart rate, and pulsed oxygen saturation were recorded before and after STST. The STST measured the submaximal performance. Thirtyeight healthy children were recruited (8-9 years: $n=19$ and 10-11 years: $n=19)$. After the STST, the perceived exertion increased with or without a facemask (8-9 years group: $+1[0.6 ; 1.4]$ and $+1.6[1.0 ; 2.1]-10-11$ years group: $+1.3[0.7$; $1.8]$ and $+1.9[1.3 ; 2.6])$ and it was higher with the facemask. The difference between the two conditions in perceived exertion was not clinically relevant in any group (mBorgf: 0.56 pts and 0.68 pts, respectively). The different domains of dyspnea assessed with Dalhousie scale were not influenced by the facemask. The submaximal performance measured by the STST was not changed by the mask whatever the age group. The cardio-respiratory demand was not clinically modified.

Conclusion: The surgical facemask had no impact on dyspnea, cardiorespiratory parameters, and exercise performance during a short submaximal exercise in healthy children.

\section{What is Known:}

- The use of facemask has been associated with breathing difficulties during physical activities in adults.

- Before the COVID-19 pandemic, the facemasks were not recommended during exercise in children and the consequences have been poorly investigated.

What is New:

- The different domains of dyspnea assessed with Dalhousie scale, the cardiac demand, and the submaximal performance measured by the STST were not changed by the facemask whatever the age group in children.
\end{abstract}

Keywords Mask · COVID-19 · Exercise capacity · Children

$\begin{array}{ll}\text { Abbreviations } \\ \text { HR } & \text { Heart rate } \\ \text { mBorg } & \text { Modified Borg scale } \\ \text { SpO2 } & \text { Pulsed oxygen saturation } \\ \text { STST } & \text { 1-Min sit-to-stand tests }\end{array}$

Communicated by Peter de Winter

Gregory Reychler

gregory.reychler@saintluc.uclouvain.be

Extended author information available on the last page of the article

\section{Introduction}

During the pandemic of the coronavirus disease 2019 (COVID-19), the wide use of facemask among the general community has been widely recommended to reduce the spread of virus-loaded respiratory droplets $[1,2]$. This measure was shown effective in reducing the transmission of SARS-CoV-2 [3]. Indeed, respiratory droplets are easily transmitted during activities of daily living $[2,4-6]$ and physical activities even increase the level of transmission [7].

As asymptomatic cases are common in children [8,9], their role in virus circulation and new COVID-19 case development has been questioned. Moreover, some debate 
quickly appeared about the need of wearing a facemask in children due to their low risk of developing the COVID-19 and the tendency to solve spontaneously [8]. The World Health Organization and United Nations International Children's Emergency Fund did not adjudicate on the use of masks for children during their common activities as in the classroom. Despite these uncertainties, many children wore facemasks [10].

Facemask has been associated with breathing difficulties in adults $[11,12]$, principally during physical activities [13]. Only a few data associated to wearability of facemask during exercise are available in children [14, 15].

The aims of the study were to evaluate the effect of wearing a facemask on perceived exertion (primary aim), dyspnea, physical performance, and cardiorespiratory response during a submaximal exercise test in children aged from 8 to 12 years. We hypothesized to observe similar findings than those found in adults.

\section{Material and methods}

\section{Subjects}

Healthy children were consecutively recruited on written parent request from one elementary school in Brussels (Belgium) during the last week of June 2021. The only inclusion criteria were (1) to be aged between 8 and 12 years old and (2) the participation in the national physical education program at school for which an annual medical investigation is required in Belgium. Eligible children had to be free of physical activity at least $1 \mathrm{~h}$ before the beginning of the experiments. The exclusion criteria were diagnosis of chronic lung, cardiac or neuromuscular disease, and motor disability based on parents' answer to standardized questions, or to be overweight (body mass index (BMI) higher than the 85th percentile for children of the same age and sex).

The study has been approved by the local Ethics Committee from Cliniques universitaires Saint-Luc and Université Catholique de Louvain in Brussels (amended in June from B4032020000121) and written informed consent was obtained from both parents and children.

\section{Study design}

It was a prospective controlled study. Each subject performed two 1-min sit-to-stand tests (STST) separated from each other by $1 \mathrm{~h}$ on the same day. One STST was performed while the children were wearing a surgical facemask whereas the other STST was performed without any facemask (control).
The order of these two STST was randomly assigned using www.randomizer.org. The children were familiarized with the STST by performing a training test at least $1 \mathrm{~h}$ before, to eliminate the learning effect. The surgical facemask was a standard 3-ply disposable facemask CE-marked that complied with the European standard EN 14,683:2019 Type I (Kimberly Clark, Machelen, Belgium).

\section{STST}

The STST was performed with a standard chair without arm rest [16]. Children put their hands on their hips, and they had to stand up and sit down completely as many times as possible during $1 \mathrm{~min}$. They could not use their arms as support. Standardized instructions were given to the children before the test. No encouragements were provided during the test. If needed, rest periods were permitted during the STST, with no interruption of the countdown timer. This exercise can be considered submaximal effort and it reflects the activities of daily life. This test was valid and reliable in children [16].

\section{Measurement of outcomes}

The demographic data were collected before the test. The perceived exertion (primary outcome) and dyspnea were rated according to the modified Borg scale (mBorg) [17] and Dalhousie scale [18] before and at the end of the STST. In the condition with the surgical facemask, dyspnea and perceived exertion collected before the STST were realized after the facemask was worn for at least $5 \mathrm{~min}$.

The mBorg ranges from zero (no perceived exertion) to ten (maximal exertion). The Dalhousie scales assess three dyspnea constructs with a sequence of pictures depicting and rating chest tightness, throat closure, and breathing effort. It includes another pictorial scale that depicts leg exertion/fatigue. The pictorial scale was explained before the test. Both scales were valid $[18,19]$ and reliable in children $[18,20]$.

The cardio-respiratory parameters (heart rate (HR), pulsed oxygen saturation ( $\mathrm{SpO} 2)$ ) were recorded before (i), at the end (f), and at 1' post-effort (r). The $\mathrm{HR}$ and $\mathrm{SpO}_{2}$ values were measured with a finger pulse oximeter (Onyx, NONIN, USA). The HR was expressed in absolute value and in percentage of theoretical maximal HR with the specific equation for children $[210-(0.65 \times$ age $)]$. The cardio-respiratory demand was assessed by the change in these outcomes between the initial and the final values and between the final and rest values expressed in absolute values for $\mathrm{SpO} 2$ and relative values for HR. 
The number of repetitions during STST was recorded and expressed in absolute values. Only completed movements were recorded.

\section{Statistical analyses}

The sample size was calculated based on an estimated difference $( \pm S D)$ of 1 and a standard deviation of 1.1 point in the modified Borg scale between the two conditions [21] with a power of $80 \%$ and an alpha level of 0.05 . In total, we aimed to recruit 38 participants to assess our hypothesis independently in children aged $8-9$ years $(n=19)$ as well as in children aged $10-11$ years $(n=19)$.

Data were analyzed using SPSS 27.0 (IBM software, Armonk, NY). Normality of data was verified with the Kolmogorov-Smirnov test. Descriptive analysis was performed for all data. They were presented as mean $( \pm S D)$ or median (interquartile range) according to the normality of the distribution.

All comparisons were tested with paired Student's $T$, Wilcoxon, or Mann-Whitney tests as appropriate. A $p$ value $\leq 0.05$ was considered statistically significant.

\section{Results}

Sixty-three children were assessed for eligibility. Two children were excluded for medical reasons, one refused to participate, and the others did not bring the acceptance from the parents. Thirty-eight healthy children were recruited in this study (8-9 years: $n=19$ and $10-11$ years: $n=19$ ). All but one in the 8-9 years group completed the two tests. This child was sick the day of the experiment. Demographic data of the children are highlighted in Table 1 . All but one child had a final HR lower than $80 \%$ of the predicted value that confirms the submaximal effort.

All children had a zero score on the mBorg at rest, before the exercise, with or without the facemask. After the STST, the perceived exertion increased in the two conditions (facemask and control) in the two age groups (8-9 years group: +1 [95\%CI: $0.6 ; 1.4] ; p<0.001$ and $+1.6[95 \%$ CI: $1.0 ; 2.1] ; p<0.001-10-11$ years group:

Table 1 Demographics characteristics of the children in each of the two age groups

\begin{tabular}{lll}
\hline Variables & $8-9$ years & $10-11$ years \\
\hline Age (years) & $8.5(8-9)$ & $10(10-11)$ \\
Gender $(\mathrm{M} / \mathrm{F})$ & $12 / 6$ & $4 / 15$ \\
Weight $(\mathrm{kg})$ & $30.2 \pm 6.0$ & $39.2 \pm 8.0$ \\
Height $(\mathrm{m})$ & $1.39(1.17-1.46)$ & $1.45(1.38-1.66)$ \\
\hline
\end{tabular}

Data are expressed as $n$, mean $\pm \mathrm{SD}$, or median (min-max)
+1.3 [95\%CI: $0.7 ; 1.8$ ]; $p<0.001$ and +1.9 [95\%CI: 1.3 ; 2.6]; $p<0.001$ ). The perceived exertion was higher with the facemask at the end of the exercise even if the mean difference between the two conditions was not clinically

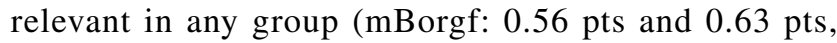
respectively).

By contrast, the different domains of dyspnea assessed with Dalhousie scale were not influenced by the facemask. Only the breathing effort domain was perceived as higher with the facemask when compared to the control condition but only for the 10-11 years group. Sixty-five percent of the children increased the perception of the breathing effort with the facemask by at least one step in the two groups. However, the domains throat closure, chest tightness, and leg exertion of the Dalhousie scale were statistically not different with or without the mask.

The cardiac demand was not changed by the mask whatever the age group. Indeed, the change in HR during effort and the recovery of HR after the test were similar between both STST (Tables 2 and 3) meaning that the mask had no effect on the HR adaptation during this effort.

The submaximal performance measured by the STST was not different when the children wore the mask or not. However, we noted that $27 \%$ and $42 \%$ of the children from the 8-9 and 10-11 years groups clinically reduced their physical performance (difference in STST >2) when they wore the facemask, respectively.

When the differences between the two conditions in the change in the cardio-respiratory outcomes during the test and in the number of sit-to-stand were compared between the 8-9 years group and the 10-11 years group, they were similar (all $p$ values $>0.05$ ).

\section{Discussion}

The main finding of the present study is that the surgical facemask increases the perceived exertion in children without modifying the different components of dyspnea during a submaximal effort. Moreover, neither the cardiorespiratory parameters nor the performances were altered when wearing a facemask during an exercise of moderate intensity. In the end, the facemask did not prevent the children from performing a short submaximal exercise.

The perceived exertion and the cardio-respiratory parameters were not modified by a facemask at rest. These findings are in agreement with the results of Li et al. who previously observed that the discomfort associated with the use of a surgical facemask was not impacted during rest periods [22]. Moreover, findings from a recent study with a similar design but conducted in adults highlighted that the surgical facemask had no immediate effect on $\mathrm{HR}, \mathrm{SpO} 2$, or dyspnea [23]. 
Table 2 Results of the two STST in children aged $8-9$ years

\begin{tabular}{llllr}
\hline Variables & With facemask & Control & Mean difference [95\%CI] & $p$ value \\
\hline Before the test & & & & \\
mBorgi (pts) & $0(0-0)$ & $0(0-0)$ & & $>0.99$ \\
Breathing effort & $1(1-2)$ & $1(1-2)$ & & $>0.99$ \\
Chest tightness & $1(1-2)$ & $1(1-2)$ & & $>0.99$ \\
Throat closure & $1(1-2)$ & $1(1-2)$ & & $>0.99$ \\
HRi (bpm) & $95(70-115)$ & $87(73-111)$ & $-4.4[-11.7 ; 2.9]$ & 0.28 \\
SpO2i (\%) & $98(97-100)$ & $99(98-99)$ & $-0.28[-0.51 ;-0.05]$ & 0.025 \\
After the test & & & & \\
mBorgf (pts) & $2(0-4)$ & $0.5(0-3)$ & $-0.56[-0.85 ;-0.26]$ & 0.004 \\
Breathing effort & $1.5(1-3)$ & $1(1-2)$ & $-0.33[-0.75 ;-0.08]$ & 0.11 \\
Chest tightness & $1(1-3)$ & $1(1-2)$ & $-0.11[0.27 ;-0.49]$ & 0.53 \\
Throat closure & $1(1-2)$ & $1(1-2)$ & $-0.11[-0.40 ; 0.18]$ & 0.41 \\
Leg exertion & $1(1-3)$ & $1(1-2)$ & $0.0[-0.30 ; 0.30]$ & $>0.99$ \\
Delta HRi-f $(\%)$ & $30.1 \pm 18.4$ & $31.0 \pm 20.2$ & $1.3[-14.0 ; 16.7]$ & 0.86 \\
HRf $(\%$ pred) & $60.1 \pm 10.7$ & $57.3 \pm 6.8$ & $-2.8[-7.5 ; 1.9]$ & 0.23 \\
SpO2f (\%) & $98(97-100)$ & $98(96-100)$ & $-0.11[-0.59 ; 0.37]$ & 0.63 \\
Delta HRf-r $(\%)$ & $-21.0 \pm 10.3$ & $-23.0 \pm 13.1$ & $-1.9[-10.6 ; 6.8]$ & 0.65 \\
STST $(n)$ & $42.5(30-63)$ & $40.5(22-63)$ & $-3.5[-7.88 ; 0.88]$ & 0.060 \\
\hline Da & & &
\end{tabular}

Data are expressed as mean $\pm \mathrm{SD}$, or median (min-max)

mBorg modified Borg scale, $H R$ heart rate, SpO2 pulsed oxygen saturation, $i$ initial, $f$ final, $p t s$ points, $S T S T$ sit-to-stand test
To our knowledge, this is the first study performed in children that evaluated the effect of a facemask during a 1-min submaximal exercise test that reflects the physical level of daily life activities. This study showed that the facemask increased the perceived exertion during the STST in children even if this difference is not clinically relevant. Indeed,
Table 3 Results of the two matched tests in children from the group 10-11 years

\begin{tabular}{llllc}
\hline Variables & With facemask & Control & Mean difference $[95 \% \mathrm{CI}]$ & $p$ value \\
\hline Before the test & & & & $>0.99$ \\
mBorgi (pts) & $0(0-0)$ & $0(0-0)$ & & $>0.99$ \\
Breathing effort & $1(1-5)$ & $1(1-5)$ & & $>0.99$ \\
Chest tightness & $1(1-5)$ & $1(1-5)$ & & $>0.99$ \\
Throat closure & $1(1-3)$ & $1(1-3)$ & & 0.39 \\
HRi (bpm) & $88(76-119)$ & $87(69-115)$ & $-3.0[-8.5 ; 2.5]$ & 0.52 \\
SpO2i (\%) & $98(97-100)$ & $98(97-100)$ & $-0.50[-0.69 ; 0.58]$ & \\
After the test & & & & 0.021 \\
mBorgf (pts) & $2(0.5-6)$ & $0.5(0-4)$ & $-0.63[-1.11 ;-0.15]$ & 0.001 \\
Breathing effort & $2(1-6)$ & $1(1-3)$ & $-0.79[-1.17 ;-0.41]$ & 0.07 \\
Chest tightness & $1(1-6)$ & $1(1-3)$ & $-0.47[-0.97 ; 0.02]$ & 0.102 \\
Throat closure & $1(1-3)$ & $1(1-3)$ & $-0.21[-0.47 ;-0.05]$ & 0.070 \\
Leg exertion & $2(1-5)$ & $1(1-3)$ & $-0.42[-0.88 ; 0.04]$ & 0.055 \\
Delta HRi-f (bpm) & $46.7 \pm 19.9$ & $54.4 \pm 21.4$ & $7.8[-0.2 ; 15.7]$ & 0.38 \\
HRf $(\%$ pred) & $65.5 \pm 6.6$ & $66.9 \pm 7.1$ & $1.4[-1.8 ; 4.5]$ & 0.13 \\
SpO2f (\%) & $98(97-99)$ & $98(94-100)$ & $-0.37[-0.86 ; 0.12]$ & 0.51 \\
Delta HRf-r (\%) & $-27.4 \pm 8.2$ & $-26.0 \pm 12.4$ & $1.4[-2.9 ; 5.7]$ & 0.34 \\
STST $(n)$ & $39(22-46)$ & $38(31-54)$ & $1.2[-1.1 ; 3.5]$ & \\
\hline
\end{tabular}

Data are expressed as mean $\pm \mathrm{SD}$, or median (min-max)

mBorg modified Borg scale, HR heart rate, SpO2 pulsed oxygen saturation, $i$ initial, $f$ final, pts points, STST sit-to-stand test 
the mean difference is lower than the minimal clinically important difference determined with the modified Borg scale (1 point). This was also demonstrated in other studies conducted in adults during an exercise of mild to moderate intensity $(9,16,18,19)$. This feeling was demonstrated to progressively increase proportionally to the exercise intensity in adults [24]. This higher perceived exertion at elevated exercise intensity is probably related to the increase in inspiratory and expiratory resistances that were previously highlighted $[24,25]$. Similarly, there was a significant reduction in running time with facemasks in trained children, at the maximum performance level [26].

The different domains of dyspnea assessed by the Dalhousie scale were low and, contrarily to the perceived exertion, not modified by the facemask in children. At rest, the different domains of the scale hovered around 1 point which was similar to the resting values found in the validation study of the Dalhousie scale in children of similar age [18]. The values of dyspnea (2.2) and perceived leg exertion (2.6) that we observed at the end of the STST correspond to values associated with peak work capacities lower than $60 \%$ [18] confirming the submaximal intensity of this test. A significant increase in breathing effort due to the surgical facemask was only noted in older children, although this difference seems clinically not relevant for the authors $(<1$ interval on the scale) and not different between the two age groups. Two hypotheses are that the older children are closer to the adult physiology and mainly the girls that dominated in this age group, and that they performed this exercise at a higher intensity than the younger ones as suggested by the greater percentage of maximal HR obtained in older children compared to the younger ones. However, a clinical change in this perception concerned more than $50 \%$ of the investigated children whatever the age of the children. The cardiac demands of the two groups were in the same range than the ones from previous studies (change in HR between 50 and 60\% [16, 27] ).

The median number of STST repetitions was around 40 repetitions. This is close to values found by other studies in children $[16,27]$ As expected, owning to the inverse relationship between height and the number of repetitions, the younger children performed more repetitions than the older ones. Indeed, the smaller children have a shorter displacement to perform than the older ones due to the chair height. That also generates a smaller effort as highlighted by the difference in HR change between the two age groups.

The main limitations of this study are the selected exercise and the environmental conditions. To extrapolate our results to a longer exercise or an exercise with a maximal intensity level, or in different environmental conditions, is hazardous. The physical performance could be differently influenced depending on the intensity of the exercise or its duration, or the ambient air temperature and humidity [28, 29]. This is due to the humidity level from the exhaled air that increase either with exercise intensity and duration or with the climate, and that changes the resistance [29]. In Belgium, the climate is temperate and maritime. Moreover, the study was performed in June and the environmental conditions change during the year. The STST does not reflect sporting activities where the duration and the intensity of the effort are different. Our results have also to be extrapolated with caution to other facemasks as the cloth facemask [23]. The powers of filtration or absolute humidity inside these facemask [22], and then the related resistances can differ. The intensity of the exercise was lower than a maximal effort as highlighted by the mean heart rate around $60 \%$ at the end of the test.

In conclusion, this study highlights that a surgical facemask had no impact on dyspnea, cardiorespiratory parameters, and exercise performance during a 1-min submaximal exercise test in healthy children. Even if the perceived exertion was slightly increased with a facemask, this is not clinically relevant. Based on this, it seems reasonable to reassure the parents about the negative perception about wearing a facemask during submaximal effort.

Acknowledgements The authors thank Ambre Van Israel who contributes to the measurements.

Authors' contributions Gregory Reychler: conceptualization; methodology; formal analysis; investigation; writing-original draft; supervision, Marie Standaert: investigation; writing-review and editing, Nicolas Audag: methodology; writing-review and editing, Annie Robert: methodology; analysis; writing-review and editing, Gilles Caty: methodology; writing-review and editing, William Poncin: methodology; analysis; writing-review and editing.

Funding Gregory Reychler and William Poncin received a grant from the Institut de Recherche Expérimentale et Clinique (Université Catholique de Louvain, Brussels, Belgium).

Availability of data material On request.

\section{Declarations}

Ethics approval The study has been approved by the local Ethics Committee from Cliniques universitaires Saint-Luc and Université Catholique de Louvain in Brussels (amended in June from B4032020000121).

Consent to participate A written informed consent was obtained from both parents and children.

Consent for publication Not applicable.

Competing interests The authors declare no competing interests.

\section{References}

1. Matuschek C, Moll F, Fangerau H, Fischer JC, Zänker K, van Griensven $\mathrm{M}$ et al (2020) Face masks: benefits and risks during the COVID-19 crisis. Eur J Med Res 25(1):32 
2. Wang Y, Xu G, Huang YW (2020) Modeling the load of SARSCoV-2 virus in human expelled particles during coughing and speaking. PLoS One 15(10):e0241539

3. Chu DK, Akl EA, Duda S, Solo K, Yaacoub S, Schunemann HJ et al (2020) Physical distancing, face masks, and eye protection to prevent person-to-person transmission of SARS-CoV-2 and COVID-19: a systematic review and meta-analysis. Lancet 395(10242):1973-1987

4. Scheuch G (2020) Breathing is enough: for the spread of influenza virus and SARS-CoV-2 by breathing only. J Aerosol Med Pulm Drug Deliv 33(4):230-234

5. Anfinrud P, Stadnytskyi V, Bax CE, Bax A (2020) Visualizing speech-generated oral fluid droplets with laser light scattering. $\mathrm{N}$ Engl J Med 382(21):2061-2063

6. Stadnytskyi V, Bax CE, Bax A, Anfinrud P (2020) The airborne lifetime of small speech droplets and their potential importance in SARS-CoV-2 transmission. Proc Natl Acad Sci U S A 117(22):11875-11877

7. Blocken B, Malizia F, Van Druenen T, Marchal T (2021) Towards aerodynamically equivalent COVID19 $1.5 \mathrm{~m}$ social distancing for walking and running. 12

8. Dong Y, Mo X, Hu Y, Qi X, Jiang F, Jiang Z et al (2020) Epidemiology of COVID-19 among children in China. Pediatrics 145(6)

9. Lu X, Zhang L, Du H, Zhang J, Li YY, Qu J et al (2020) SARSCoV-2 infection in children. N Engl J Med 382(17):1663-1665

10. Mickells GE, Figueroa J, West KW, Wood A, McElhanon BO (2021) Adherence to masking requirement during the COVID19 pandemic by early elementary school children. J Sch Health 91(7):555-561

11. Jefferson T, Del Mar CB, Dooley L, Ferroni E, Al-Ansary LA, Bawazeer GA et al (2020) Physical interventions to interrupt or reduce the spread of respiratory viruses. Cochrane Database Syst Rev 11:Cd006207

12. Canini L, Andréoletti L, Ferrari P, D'Angelo R, Blanchon T, Lemaitre $M$ et al (2010) Surgical mask to prevent influenza transmission in households: a cluster randomized trial. PLoS One 5(11):e13998

13. Mask use in the context of COVID-19: interim guidance (2020) In: World Health Organization. [Internet]. Available from: https:// www.apps.who.int/iris/handle/10665/337199

14. Eberhart M, Orthaber S, Kerbl R (2021) The impact of face masks on children-a mini review. Acta Paediatr 110(6):1778-1783

15. Lubrano R, Bloise S, Testa A, Marcellino A, Dilillo A, Mallardo $S$ et al (2021) Assessment of respiratory function in infants and young children wearing face masks during the COVID-19 pandemic. JAMA Netw Open 4(3):e210414

16. Reychler G, Audag N, Mestre NM, Caty G (2019) Assessment of validity and reliability of the 1-minute sit-to-stand test to measure the heart rate response to exercise in healthy children. JAMA Pediatr 173(7):692-693
17. Borg E, Borg G, Larsson K, Letzter M, Sundblad BM (2010) An index for breathlessness and leg fatigue. Scand J Med Sci Sports 20(4):644-650

18. Pianosi PT, Huebner M, Zhang Z, McGrath PJ (2014) Dalhousie dyspnea and perceived exertion scales: psychophysical properties in children and adolescents. Respir Physiol Neurobiol 199:34-40

19. Hommerding PX, Donadio MV, Paim TF, Marostica PJ (2010) The Borg scale is accurate in children and adolescents older than 9 years with cystic fibrosis. Respir Care 55(6):729-733

20. Pfeiffer KA, Pivarnik JM, Womack CJ, Reeves MJ, Malina RM (2002) Reliability and validity of the Borg and OMNI rating of perceived exertion scales in adolescent girls. Med Sci Sports Exerc 34(12):2057-2061

21. Person E, Lemercier C, Royer A, Reychler G (2018) Effect of a surgical mask on six minute walking distance. Rev Mal Respir 35(3):264-268

22. Li Y, Tokura H, Guo YP, Wong AS, Wong T, Chung J et al (2005) Effects of wearing N95 and surgical facemasks on heart rate, thermal stress and subjective sensations. Int Arch Occup Environ Health 78(6):501-509

23. Reychler G, Straeten CV, Schalkwijk A, Poncin W (2021) Effects of surgical and cloth facemasks during a submaximal exercise test in healthy adults. Respir Med 186:106530

24. Mapelli M, Salvioni E, De Martino F, Mattavelli I, Gugliandolo $P$, Vignati $C$ et al (2021) You can leave your mask on: effects on cardiopulmonary parameters of different airway protection masks at rest and during maximal exercise. Eur Respir J

25. Fikenzer S, Uhe T, Lavall D, Rudolph U, Falz R, Busse M et al (2020) Effects of surgical and FFP2/N95 face masks on cardiopulmonary exercise capacity. Clin Res Cardiol 109(12):1522-1530

26. Schulte-Korne B, Hollmann W, Vassiliadis A, Predel HG (2021) Effects of surgical face masks on exercise performance and perceived exertion of exercise in well-trained healthy boys. Wien Med Wochenschr

27. Reychler G, Pincin L, Audag N, Poncin W, Caty G (2020) Oneminute sit-to-stand test as an alternative tool to assess the quadriceps muscle strength in children. Respir Med Res 78:100777

28. Meyer JP, Hery M, Herrault J, Hubert G, Francois D, Hecht G et al (1997) Field study of subjective assessment of negative pressure half-masks. Influence of the work conditions on comfort and efficiency. Appl Ergon 28(5-6):331-338

29. Nisar M, Spence DP, West D, Haycock J, Jones Y, Walshaw MJ et al (1992) A mask to modify inspired air temperature and humidity and its effect on exercise induced asthma. Thorax 47(6):446-450

Publisher's Note Springer Nature remains neutral with regard to jurisdictional claims in published maps and institutional affiliations. 


\section{Authors and Affiliations}

\section{Gregory Reychler ${ }^{1,2,3,4}$ (D) Marie Standaert ${ }^{4} \cdot$ Nicolas Audag ${ }^{1,2,3,4} \cdot$ Gilles Caty $^{5} \cdot$ Annie Robert $^{6} \cdot$ William Poncin $^{1,2,3,4}$}

Marie Standaert

marie.standaert@vinci.be

Nicolas Audag

nicolas.audag@saintluc.uclouvain.be

Gilles Caty

gilles.caty@chwapi.be

Annie Robert

annie.robert@saintluc.uclouvain.be

William Poncin

william.poncin@saintluc.uclouvain.be

1 Institut de Recherche Expérimentale Et Clinique (IREC), Pôle de Pneumologie, ORL Et Dermatologie,

Université Catholique de Louvain, Avenue Hippocrate 55, 1200 Brussels, Belgium
2 Service de Pneumologie, Cliniques Universitaires Saint-Luc, Avenue Hippocrate 10, 1200 Brussels, Belgium

3 Secteur de Kinésithérapie Et Ergothérapie, Cliniques Universitaires Saint-Luc, Avenue Hippocrate 10, 1200 Brussels, Belgium

4 Département de Kinésithérapie, Haute École Léonard de Vinci, Parnasse-ISEI, Brussels, Belgium

5 Service de Médecine Physique, Centre Hospitalier Wallonie Picarde (CHWAPI), Tournai, Belgium

6 Pôle Epidémiologie Et Biostatistique, Institut de Recherche Expérimentale Et Clinique, UCLouvain, Brussels, Belgium 